\title{
Intraperitoneal LPS amplifies portal hypertension in rat liver fibrosis
}

\author{
Christian J Steib ${ }^{1}$, Anna C Hartmann ${ }^{1}$, Christoph v Hesler ${ }^{1}$, Andreas Benesic ${ }^{1}$, Martin Hennenberg ${ }^{2}$, Manfred Bilzer ${ }^{3}$ \\ and Alexander L Gerbes ${ }^{1}$
}

Recent studies have shown that the risk of variceal bleeding in patients with liver cirrhosis increases with infections such as spontaneous bacterial peritonitis (SBP). In this study, we hypothesized that pretreatment with intraperitoneal LPS may escalate portal hypertension. In fibrotic livers ( 4 weeks after bile duct ligation, BDL), the activation of Kupffer cells (KCs) by zymosan $(150 \mu \mathrm{g} / \mathrm{ml})$ in the isolated non-recirculating liver perfusion system resulted in a transient increase in portal perfusion pressure. Pretreatment with intraperitoneal LPS (1 mg/kg body weight (b.w.) for $3 \mathrm{~h}$ ) increased basal portal perfusion pressure, and prolonged the zymosan-induced increase from transient to a long-lasting increase that was sustained until the end of the experiments in BDL but not in sham-operated animals. Pretreatment with gadolinium chloride (10 mg/kg b.w.), MK-886 (0.6 mg/kg b.w.), Ly171883 $(20 \mu \mathrm{M})$ or BM $13.177(20 \mu \mathrm{M})$ reduced the maximal and long-lasting pressure increase in $\mathrm{BDL}$ animals by approximately $50-60 \%$. The change in portal perfusion pressure was paralleled by a long-lasting production of cysteinyl leukotriene (Cys-LT) and thromboxane (TX) after LPS pretreatment. However, the response to vasoconstrictors was not altered by intraperitoneal LPS. Western blot analyses showed an increased Toll-like receptor (TLR)4 and MyD88 expression after LPS pretreatment. In vivo experiments confirmed that intraperitoneal LPS increased basal portal pressure, and extended the portal pressure increase produced by intraportal zymosan or by LPS infusion. In conclusion, upregulation of TLR4 and MyD88 expression in fibrotic livers confers hypersensitivity to LPS. This may lead to escalation of portal hypertension by production of TX and Cys-LT after endotoxin-induced KC activation. Therefore, LT inhibitors may represent a promising treatment option in addition to early administration of antibiotics in SBP.

Laboratory Investigation (2010) 90, 1024-1032; doi:10.1038/labinvest.2010.60; published online 8 March 2010

KEYWORDS: portal pressure; bile duct ligation; Kupffer cell; thromboxane; leukotriene; TLR4

Liver cirrhosis is associated with the development of hyperdynamic circulation and portal hypertension. ${ }^{1}$ The increase in portal pressure is triggered by persistent splanchnic vasodilation and increased intrahepatic vascular resistance. Molecular changes in the cirrhotic liver include alterations in the generation and degradation of vasodilators and vasoconstrictors, as well as in changes in cellular responses to the same. ${ }^{2-4}$ Hepatic vascular tone changes are caused by an increase in vasoconstrictors such as thromboxane (TX) $\mathrm{A}_{2}{ }^{5,6}$ or cysteinyl leukotrienes (Cys-LT: leukotrine $\left.\mathrm{C}_{4}, \mathrm{D}_{4}, \mathrm{E}_{4}\right){ }^{7}$ and a decrease in vasodilators. ${ }^{8}$

Variceal bleeding is a life-threatening complication of portal hypertension. Mortality is closely related to any failure to control the initial bleed or early rebleeding, which can occur in up to $30-40 \%$ of patients. ${ }^{9}$ Variceal bleeding is closely associated with bacterial infections. ${ }^{10-13}$ In patients with cirrhosis, the risk of variceal bleeding increases with infections such as spontaneous bacterial peritonitis (SBP). However, the pathophysiology of this phenomenon is not well understood. The phagocytosis of bacterial products in the liver is predominantly dependent on the activity of Kupffer cells (KCs), which are hepatic macrophages. ${ }^{14,15}$ Therefore, KCs could have a leading role in the regulation of infection-related variceal bleeding, as proposed previously. ${ }^{16}$ The activation of KCs is dependent on surface receptors such as Toll-like receptors (TLRs) and intracellular effectors such as MyD88.

Patients with liver cirrhosis often suffer from SBP, which is partly caused by increased gut permeability and decreased

\footnotetext{
${ }^{1}$ Department of Medicine II (Gastroenterology and Hepatology), Liver Center Munich, University of Munich—Grosshadern, Munich, Germany; ${ }^{2}$ Department of Urology, University of Munich-Grosshadern, Munich, Germany and ${ }^{3}$ Essex Pharma, Munich, Germany

Correspondence: Dr CJ Steib, MD, Klinikum Grosshadern—Department of Medicine II, Liver Center Munich (LCM), Ludwig-Maximilians University, Marchioninistrasse 15, 81377 Munich, Germany.

E-mail: christian.steib@med.uni-muenchen.de

Received 8 September 2009; revised 5 January 2010; accepted 24 January 2010
} 
bowel motility. ${ }^{17,18}$ In this study, the effects of intraperitoneal LPS on portal pressure and the involved pathways were investigated.

\section{MATERIALS AND METHODS Animal Studies}

All animals were ethically treated according to the criteria prepared by the National Academy of Sciences and published by the National Institutes of Health in addition to the legal requirements in Germany. All animal experiments were approved by the local government (Regierung von Oberbayern, Munich, Germany) and were reported to the responsible authorities every 3 months.

\section{Induction of liver fibrosis by bile duct ligation}

Male Sprague-Dawley rats (weighing 180-200 g; Charles River, Sulzfeld, Germany) were anesthetized by intraperitoneal pentobarbital injection $(50 \mathrm{mg} / \mathrm{kg}$ body weight (b.w.)). After a midline laparotomy was performed, the common bile duct was ligated twice with 3-0 silk suture and cut between the two ligations. ${ }^{6,19}$ Sham-operated rats were subjected to laparotomy without bile duct ligation (BDL). Liver perfusion with Krebs-Henseleit buffer and in vivo experiments were performed 4 weeks after the operation, as described in the following sections.

\section{In situ rat liver perfusion study}

Four weeks after sham operation or BDL, rats were anesthetized with an intraperitoneal injection of sodium pentobarbital $(50 \mathrm{mg} / \mathrm{kg}$ b.w.). After incision of the abdominal wall, blood was collected from the inferior vena cava (approximately 3-4 ml) to determine serum parameters. Thereafter, the portal vein was cannulated with a 14-G Teflon intravenous catheter, and the liver was perfused at a constant flow rate. The inferior vena cava was cannulated through the right atrium and ligated above the right renal vein. In all experiments, the livers were perfused with Krebs-Henseleit solution $\left(\mathrm{pH} 7.4,37^{\circ} \mathrm{C}\right)$ in a non-recirculating manner. The perfusion buffer was gassed with a mixture of $95 \% \mathrm{O}_{2}$ and $5 \% \mathrm{CO}_{2}$ using an oxygenator. ${ }^{6,20}$ Portal perfusion pressure was monitored continuously. The bile duct was cannulated with polyethylene tubing (PE10) to monitor bile flow in both sham-operated and non-operated animals. The liver was allowed to stabilize for $25 \mathrm{~min}$ before any substances were added. During this initial 25-min stabilization period, the criteria for liver viability were stable perfusion pressures and LDH efflux rates $<5 \mathrm{mU} / \mathrm{min} \times$ gram liver. $\mathrm{LDH}$ activity was analyzed as reported previously. Even if one of the criteria for viability was not satisfied, the experiment was discarded.

\section{Intraperitoneal LPS administration and KC activation}

All the following experiments were conducted with and without additional intraperitoneal LPS administration for $3 \mathrm{~h}$ (Escherichia coli 026:B6, $1 \mathrm{mg} / \mathrm{kg}$ b.w. dissolved in $\mathrm{NaCl}$ $0.9 \%)$. KCs were activated by the infusion of cell wall particles from Saccharomyces cerevisiae (zymosan A, $n=5$ ). Zymosan was prepared and infused as described previously (150 $\mu \mathrm{g} / \mathrm{ml}, 40-46 \mathrm{~min}$ after starting perfusion experiments). ${ }^{6}$ In control experiments, the livers were perfused with KrebsHenseleit buffer in addition to the solvent DMSO $(22 \mathrm{mM}$, $30-60 \mathrm{~min}, \quad n=4)$ after zymosan infusion $(150 \mu \mathrm{g} / \mathrm{ml}$, 40-46 min after starting the perfusion experiments). To block KCs, a pretreatment with gadolinium chloride $\left(\mathrm{GdCl}_{3}\right.$, $10 \mathrm{mg} / \mathrm{kg}$ b.w., 48 and $24 \mathrm{~h}$ before experiments) was performed in addition to LPS administration and after KC activation $(n=5) .^{21}$ The LT receptor antagonist Ly171883 $(20 \mu \mathrm{M}, 30-60 \mathrm{~min}, n=5)$ and the TX receptor antagonist BM $13.177(20 \mu \mathrm{M}, 30-60 \mathrm{~min}, n=5)$ were given in addition to LPS pretreatment $(1 \mathrm{mg} / \mathrm{kg}$ b.w., $3 \mathrm{~h})$ and zymosan infusion $(150 \mu \mathrm{g} / \mathrm{ml}, 40-46 \mathrm{~min})$. The LT synthase inhibitor MK$886(0.6 \mathrm{mg} / \mathrm{kg} \quad$ b.w. $)$ was administered intraperitoneally $30 \mathrm{~min}$ before starting LPS pretreatment $(1 \mathrm{mg} / \mathrm{kg}$ b.w. for $3 \mathrm{~h})$; zymosan $(150 \mu \mathrm{g} / \mathrm{ml}, 40-46 \mathrm{~min})$ was administered during liver perfusion, which started altogether $3.5 \mathrm{~h}$ after MK-886 administration.

\section{Infusion of the vasoconstrictors $T X A_{2}$ and $L T C_{4}$}

The TX $\mathrm{A}_{2}$ analog $\mathrm{U} 46619(0.1 \mu \mathrm{M}, 40-46 \mathrm{~min}, n=5)$ and $\mathrm{LTC}_{4}(20 \mathrm{nM}, 40-46 \mathrm{~min}, n=5)$ were infused with and without additional intraperitoneal LPS administration ( $1 \mathrm{mg} /$ $\mathrm{kg}$ b.w. for $3 \mathrm{~h}$, each $n=5$ ).

\section{In vivo measurement of portal pressure}

Body weight was determined directly before experiments. Surgery (4 weeks after BDL) was commenced after anesthesia using an intraperitoneal injection of sodium pentobarbital $(50 \mathrm{mg} / \mathrm{kg}$ b.w.). Arterial blood pressure was monitored continuously through a carotid catheter. Laparotomy was performed, and a PE-tube $\left(23 \mathrm{G}, 0.6 \times 30 \mathrm{~mm}^{2}\right)$ was inserted over the ileocolic vein and advanced into the confluence of the portal and splenic veins. This cannula was used for the simultaneous infusion of zymosan and the registration of portal pressure through a transducer system (Sirecust 404 from Siemens, Germany and Transducer Gabarith from Becton Dickinson, Singapore), as described previously. ${ }^{6}$ Two different bacterial products were infused in distinct experiments through the portal vein: $\beta$-glycan-rich zymosan $(3.2 \mathrm{mg} / \mathrm{min} \text {, from } 0-6 \mathrm{~min}, n=7)^{6,22}$ or LPS $(4 \mathrm{mg} / \mathrm{kg}$ b.w. $0-6 \mathrm{~min}, n=7)$. In an additional set of experiments, animals were pretreated with intraperitoneal LPS for $3 \mathrm{~h}(1 \mathrm{mg} / \mathrm{kg}$ b.w. for $3 \mathrm{~h}$, each $n=7$ ), and then zymosan or LPS was administered as stated above.

\section{Measurements of Serum Parameters}

Serum parameters were measured using an Olympus AU2700 analyzer (Olympus Germany GmbH, Hamburg, Germany), according to standard tests based on the recommendations of the International Federation of Clinical Chemistry (IFCC). 


\section{Histological Evaluation}

Liver tissues were fixed in $4 \%$ buffered formalin, dehydrated in graded ethanol and embedded in paraffin using standard procedures. Longitudinal sections of $4-\mu \mathrm{m}$ thickness were stained with hematoxylin and eosin (H\&E) and Elastica van Gieson (EvG) using standard procedures.

\section{ELISA}

The levels of $\mathrm{TXB}_{2}$ (the stable degradation product of $\mathrm{TXA}_{2}$ ) produced by the livers were quantified by measuring their release into the perfusate. The levels of $\mathrm{TXB}_{2}$ were measured in duplicate using an enzyme immunoassay (Assay Design, Ann Arbor, MI, USA). The levels of Cys-LTs $\left(\mathrm{LTC}_{4} / \mathrm{D}_{4} / \mathrm{E}_{4}\right)$ were also measured in duplicate using an enzyme immunoassay (Cayman Chemical, Ann Arbor, MI, USA). Serum immunoreactive IL-6 levels were also determined in duplicate by a rat-specific ELISA linked to horseradish peroxidase (HRP) and with a detection limit of $30 \mathrm{pg} / \mathrm{ml}$ (Biosource International, Camarillo, CA, USA). Assay specificity was confirmed and percentage recoveries were determined by serum samples with recombinant rat IL- 6 as the standard.

\section{Western Blot Analysis for TLR4 and MyD88}

Western blot analysis was performed according to standard procedures. Briefly, proteins transferred to the membrane were detected by incubation with primary antibodies (antiTLR4, anti-goat polyclonal; Santa Cruz, USA, 1:200 at $4^{\circ}$ C, overnight or anti-MyD88, anti-rabbit polyclonal, Abcam, USA, 1:500 at $4^{\circ} \mathrm{C}$, overnight), followed by incubation with an HRP-conjugated secondary antibody (Santa Cruz or GE Healthcare Life Sciences, Munich, Germany). The reactive bands were visualized using a chemiluminescent detection kit (ECL Plus, Amersham Pharmacia, Uppsala, Sweden). $\beta$-Actin was used as a control (GE Healthcare Life Sciences). The protein expression was quantified using the ImageJ software (NIH, USA).

\section{Drugs and Reagents}

Zymosan and LPS from E. coli (026:B06) were obtained from Sigma-Aldrich (Munich, Germany). Ly171883 was obtained from Calbiochem (Darmstadt, Germany). BM 13.177 was a gift from Roche (Mannheim, Germany). U46619 and LTC $_{4}$ were purchased from Cayman Chemical.

\section{Statistical Analyses}

All data are expressed as means \pm s.d. Statistical analyses of the data were performed using Mann-Whitney $U$-test for not normally distributed data. A value of $P<0.05$ was considered to be statistically significant. Statistical preparation and analyses were performed in collaboration with the Institute for Biometrics and Epidemiology of the University of Munich (IBE, Munich, Germany).

\section{RESULTS \\ Enhancement of Portal Hypertension by Preactivation with LPS}

Portal pressure of BDL rats, determined immediately before the isolated liver perfusion experiments, was elevated to $21.5 \pm 1.2 \mathrm{~cm} \mathrm{H}_{2} \mathrm{O}$, which was greater than that of shamoperated animals $\left(8.8 \pm 0.9 \mathrm{~cm} \mathrm{H}_{2} \mathrm{O}\right)$. All BDL rats had elevated values on liver function tests compared with shamoperated animals ( $\gamma$-glutamyltransferase (U/l): $21 \pm 1.3$ vs $1.3 \pm 0.4$, bilirubin $(\mathrm{mg} / \mathrm{dl}): 8.3 \pm 0.5$ vs $0.2 \pm 0.1$, aspartate aminotransferase (U/l): $98 \pm 17$ vs $45 \pm 8$ and alanine aminotransferase (U/l): $38 \pm 5 v s 34 \pm 5$ ) and the weight of the spleen had also increased (data not shown). Portal pressure in $\mathrm{BDL}$ rats was further increased by application of intraperitoneal LPS to $25.2 \pm 1.7 \mathrm{~cm} \mathrm{H}_{2} \mathrm{O}$. In contrast, pretreatment with LPS did not alter the basal portal pressure in sham-operated animals $\left(9.1 \pm 0.8 \mathrm{~cm} \mathrm{H}_{2} \mathrm{O}\right)$. Subsequent to portal pressure measurements in vivo, the livers were isolated and perfused in situ. Again, portal perfusion pressure was higher in the BDL group with additional LPS pretreatment than in the BDL-only group. Similarly, portal perfusion pressure of in situ-perfused livers was still higher in the BDL group than in the sham group (Figure 1a). The maximal zymosan-induced increase in portal perfusion pressure was higher in the BDL group with the additional LPS pretreatment than in the BDL group without LPS pretreatment, and this difference was enhanced until the end of the perfusion period (Figure 1a). In contrast, LPS pretreatment did not influence the portal perfusion pressure increase in the sham group (Figure 1a). The measurement of IL-6 in the serum showed that no IL-6 was detectable before LPS pretreatment in sham-operated animals (serum IL- $6<0.03 \mathrm{ng} / \mathrm{ml}$ ) and in BDL animals (serum IL- $6<0.03 \mathrm{ng} / \mathrm{ml}$ ). LPS pretreatment increased the IL- 6 values in both groups, ie, sham-operated animals and BDL animals, but the IL-6 level increased to a higher level in BDL animals $(3.4+0.7$ vs $12.8+2.5 \mathrm{ng} / \mathrm{ml}$, $P<0.05)$. The livers of sham-operated and BDL animals with and without LPS pretreatment were analyzed by H\&E and EvG staining to exclude morphological alterations by LPS pretreatment. Sham-operated animals pretreated with vehicle or LPS showed normal liver histology (Figure 1b). Livers from BDL rats showed typical morphological signs of these livers with proliferation of bile ducts, mixed cellular infiltration around the portal vein, isolated areas of necrosis and fibrotic alterations (Figure 1b). No morphological differences could be detected in the above-mentioned characteristics of the BDL livers pretreated with LPS compared with vehicle-treated rats. The effects on portal pressure of LPS pretreatment with subsequent zymosan infusion were reversed by a pretreatment with $\mathrm{GdCl}_{3}$ that blocked the KCs (Figure $1 \mathrm{~b}$ ).

\section{Increase in the Protein Expression of TLR4 and MyD88 by LPS Pretreatment}

The expression of TLR4 and MyD88 was analyzed by western blotting and subsequent quantification. Application of LPS in 


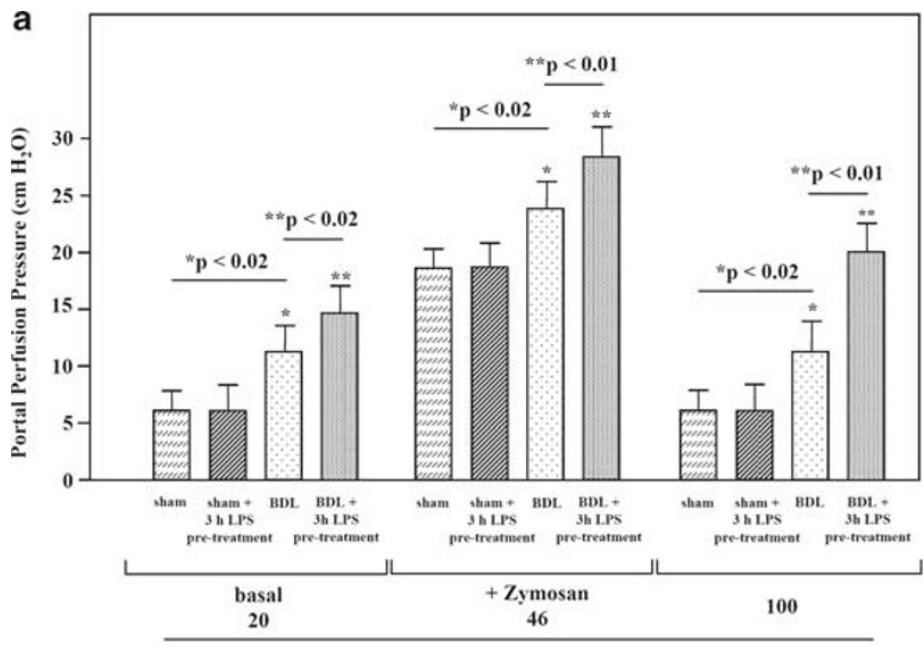
+ vehicle

C

Time Point Portal Perfusion Pressure Measurement (min)

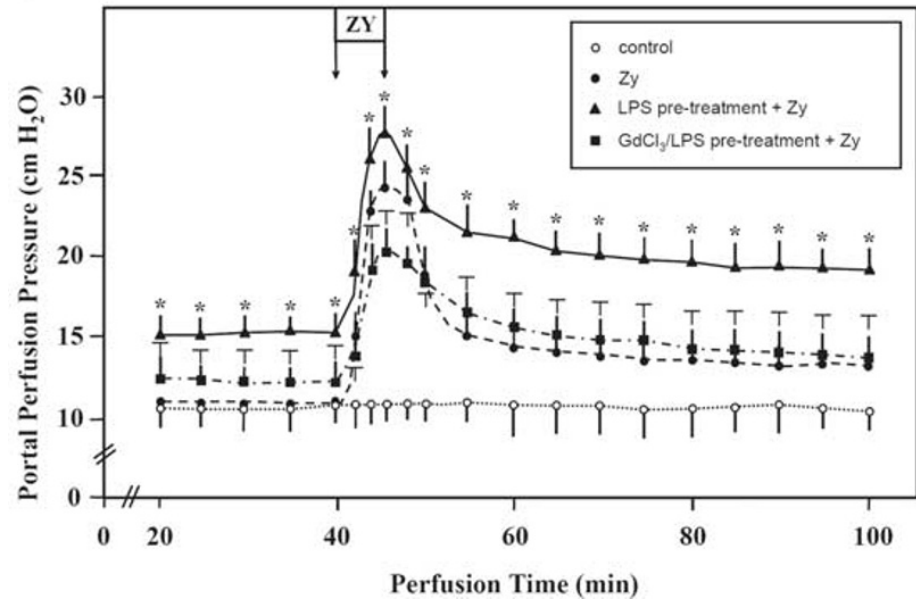

b sham
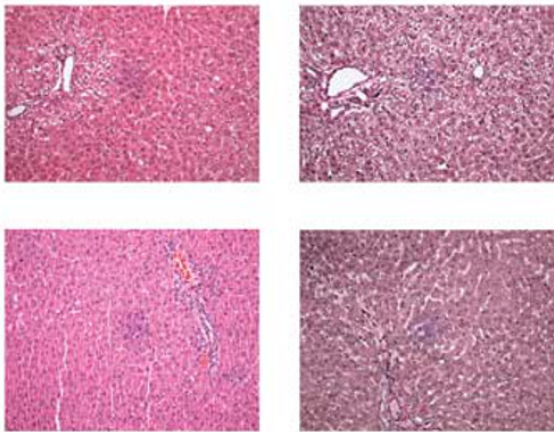

sham $+3 \mathrm{~h}$ LPS
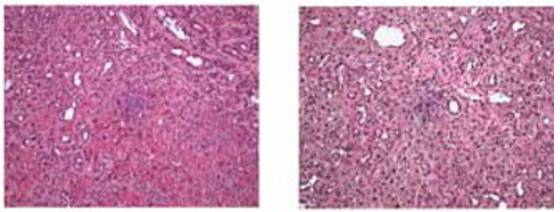

$B D L$

$+3 \mathrm{~h}$ LPS

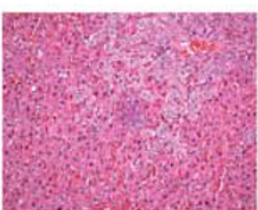

HE, 200x

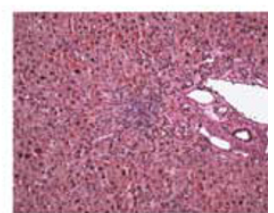

EvG, 200x

Figure 1 Intraperitoneal LPS escalates the KC-dependent portal pressure increase in fibrosis. Data are expressed as mean \pm s.d. (a) Portal perfusion pressure started at a lower basal level in sham-operated rats $(n=5)$ compared with the bile duct-ligated rats $\left({ }^{\star} P<0.02, \mathrm{BDL}\right.$ for 4 weeks, $\left.n=5\right)$. The basal portal perfusion pressure, and hence the maximal portal perfusion pressure, was further enhanced by LPS pretreatment for $3 \mathrm{~h}(1 \mathrm{mg} / \mathrm{kg}$ b.w. intraperitoneal, $n=5$ ) in animals with biliary fibrosis ( $\left.{ }^{*} P<0.01\right)$, but not in the sham-operated animals. In addition, transient portal perfusion pressure increase was extended to a long-lasting portal perfusion pressure increase in LPS-pretreated BDL rats ( ${ }^{*} P<0.01$ ), but, again, not in sham-operated animals. (b) The microscopic analysis showed normal liver histology in sham-operated rats with and without LPS pretreatment. Furthermore, the typical histological picture of BDL livers (proliferation of bile ducts, mixed cellular infiltration around the portal vein, isolated areas of necrosis, fibrotic alterations) was observed in both groups, ie, in BDL animals and in BDL animals pretreated with LPS. No morphological differences could be detected in the mentioned characteristics of the BDL livers pretreated with LPS. (c) KC activation by Zymosan A ( $, Z y, 150 \mu \mathrm{g} / \mathrm{ml}, 40-46 \mathrm{~min}, n=5)$ in BDL animals transiently increased portal perfusion pressure compared with control experiments $(0, n=5)$. These animals were additionally pretreated with $\mathrm{NaCl}(0.9 \%)$. Intraperitoneal LPS ( $1 \mathrm{mg} / \mathrm{kg}$ b.w. for $3 \mathrm{~h}, n=5)$ increased the basal, maximal and chronic portal perfusion pressures $\left({ }^{*} P<0.05\right)$. In the next experiments, BDL animals were pretreated with gadolinium chloride to block Kupffer cells ( $\square, \mathrm{GdCl}_{3}, 48$ and $24 \mathrm{~h}$ before starting experiments, $10 \mathrm{mg} / \mathrm{kg}$ b.w. intraperitoneal, $n=5$ ), were then pretreated with $\mathrm{LPS}$ ( $1 \mathrm{mg} / \mathrm{kg}$ b.w. for $3 \mathrm{~h}$, intraperitoneal), and then zymosan was infused during the isolated liver perfusion. GdCl $\mathrm{pretreatment}$ attenuated the basal, maximal and chronic portal perfusion pressure increases $\left.{ }_{\top} P<0.05\right)$.

BDL rats caused upregulation of TLR4 and MyD88 expression in the liver (Figure $2 a$ and $b$ ). This effect of LPS on hepatic TLR4 and MyD88 expression in BDL rats was prevented by $\mathrm{KC}$ blockade with $\mathrm{GdCl}_{3}$ (Figure $2 \mathrm{a}$ and $\mathrm{b}$ ).

\section{Augmented Production of the Vasoconstrictors and their Functional Relevance}

The zymosan-induced increase in portal perfusion pressure was paralleled by zymosan-induced production of the vasoconstrictor $\mathrm{TXB}_{2}$, the stable degradation product of $\mathrm{TXA}_{2}$, and of the vasoconstrictive Cys-LTs (Figure 3a and b). Although this increase was transient in the BDL group, the increase was long lasting in the LPS pretreatment group for both vasoconstrictor types $\left(\mathrm{TXB}_{2}\right.$ and $\left.\mathrm{LTC}_{4} / \mathrm{D}_{4}\right)$ (Figure $3 \mathrm{a}$ and $\mathrm{b}$ ). The initial and maximal levels of $\mathrm{TXB}_{2}$ and Cys-LTs detected in the effluent perfusate were increased by LPS pretreatment (Figure $3 \mathrm{a}$ and b). The functional relevance of these vasoconstrictors was evaluated 
by the administration of the LT receptor inhibitor Ly171883, the LT synthase inhibitor MK-886 and the TX receptor antagonist BM 13.177. The blockade of the LT receptor attenuated both the maximal immediate and the long-lasting zymosan-induced increase of portal perfusion pressure (Figure 3c). A similar result was achieved by the TX receptor antagonist BM 13.177 (Figure 3c). Both the long-lasting and the maximal immediate zymosaninduced increases of portal perfusion pressure were

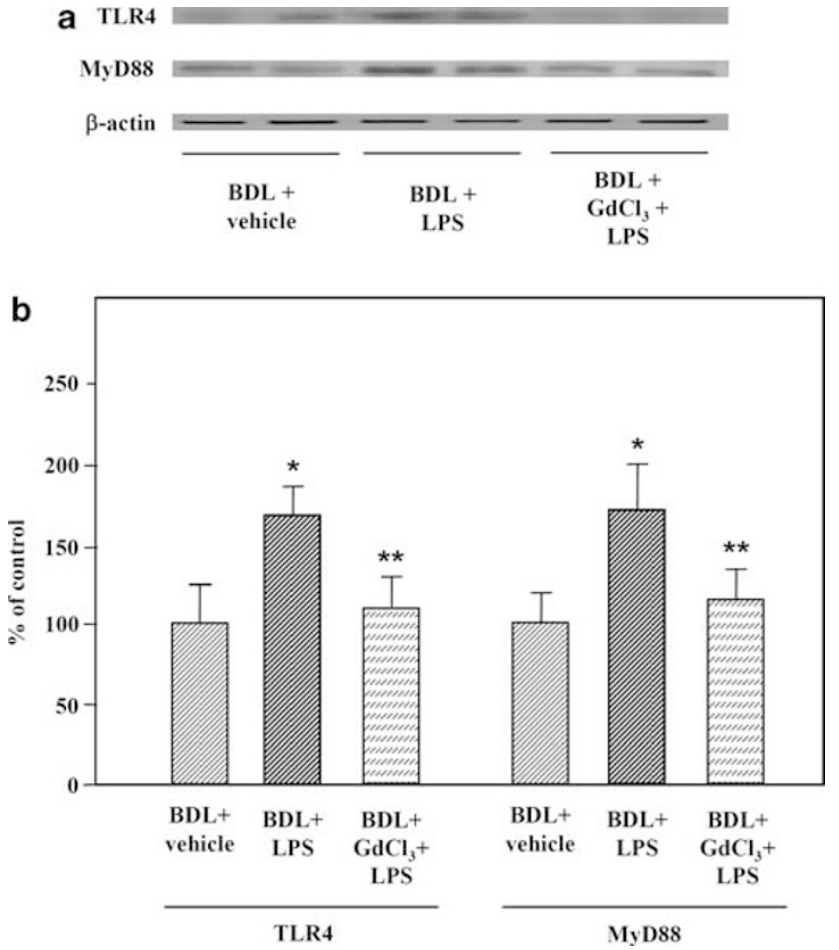

Figure 2 Increased expression of TLR4 and MyD88 after intraperitoneal LPS pretreatment. (a) The protein expression (measured by western blot) of TLR4 and MyD88 was increased in the bile duct-ligated (BDL) animals by LPS pretreatment $(1 \mathrm{mg} / \mathrm{kg}$ b.w. for $3 \mathrm{~h}$, each $n=5)$ compared with BDL animals with vehicle pretreatment $(\mathrm{NaCl} 0.9 \% 1 \mathrm{ml} / \mathrm{kg}$ b.w., $n=5)$. The augmented protein expression after LPS pretreatment in BDL animals was attenuated by additional $\mathrm{GdCl}_{3}$ pretreatment $(10 \mathrm{mg} / \mathrm{kg}$ b.w., 24 and $48 \mathrm{~h}$ before experiments, $n=5$ ). $\beta$-actin served as a control. (b) These results were confirmed by quantitative analyses by ImageJ ( $\mathrm{NIH}$, USA). attenuated by the additional infusion of BM 13.177. The intraperitoneal pretreatment over a period of $3.5 \mathrm{~h}$ with the LT synthase inhibitor MK-886 reduced the basal, maximal and long-lasting portal perfusion pressure increases. In contrast, the IL-6 concentration in the serum was not influenced by MK-886 pretreatment $(11.8+2.1$ vs $12+2.5 \mathrm{ng} / \mathrm{ml})$.

\section{Unaltered Response to the Vasoconstrictors After LPS Pretreatment}

In contrast to the production of the vasoconstrictors, which was enhanced by LPS, the responsiveness to the vasoconstrictors was not influenced by LPS pretreatment over $3 \mathrm{~h}$. The infusion of the TX analog U46619 increased the portal perfusion pressure of BDL rats transiently (Figure 4a). Neither the maximal portal perfusion pressure nor the long-lasting portal perfusion pressure was influenced by pretreatment with LPS (Figure 4a). Similar to U46619, the infusion of $\mathrm{LTC}_{4}$ also increased the portal perfusion pressure transiently (Figure $4 \mathrm{~b}$ ). The maximal portal perfusion pressure after $\mathrm{LTC}_{4}$ infusion was slightly lower than that after U46619 infusion (Figure 4a and b). Again, neither the maximal nor the long-lasting portal perfusion pressure increase caused by $\mathrm{LTC}_{4}$ was influenced by pretreatment with LPS (Figure 4b).

\section{Effects of LPS on Portal Pressure: In Vivo Measurements}

The results obtained from the isolated liver perfusions were confirmed by in vivo experiments. Portal pressure was higher in the BDL group with LPS pretreatment than in the BDL group without LPS pretreatment (Figure $5 \mathrm{a}$ and $\mathrm{b}$ ). The infusion of zymosan or LPS increased the portal pressure (Figure $5 \mathrm{a}$ and $\mathrm{b}$ ). The absolute extent in portal pressure increase after zymosan or LPS infusion was comparable in the groups with and without LPS pretreatment (Figure 5a and $\mathrm{b}$ ). However, the absolute value of maximal portal pressure was higher in the LPS pretreatment groups (Figure $5 \mathrm{a}$ and $\mathrm{b}$ ). The long-lasting zymosan- and LPS-induced increases of the portal pressure were again markedly enhanced by LPS pretreatment before intraportal LPS or zymosan infusion (Figure $5 \mathrm{a}$ and $\mathrm{b}$ ). The mean arterial pressure

Figure 3 Enhanced production of the vasoconstrictors and their functional role after intraperitoneal LPS administration in liver fibrosis. Data are expressed as mean \pm s.d. (a) The increase in portal perfusion pressure in the bile duct-ligated (BDL) animals after Zymosan A (o, Zy, 150 $\mu \mathrm{g} / \mathrm{ml}, 40-46 \mathrm{~min}, n=5)$ was paralleled by an augmented production of thromboxane $(T X) B_{2}$, the stable degradation product of thromboxane $A_{2}$. TX production was further enhanced by intraperitoneal LPS pretreatment $(\bullet, 1 \mathrm{mg} / \mathrm{kg}$ b.w. for $3 \mathrm{~h}, n=5)$ in addition to KC activation by zymosan $\left(150 \mu \mathrm{g} / \mathrm{ml}, 40-46 \mathrm{~min},{ }^{\star} P<0.05\right)$. (b) The cysteinyl leukotrienes (LTs) $C_{4} / D_{4} / E_{4}$ were produced by KC activation (o, Zy, $150 \mu \mathrm{g} / \mathrm{ml}, 40-46 \mathrm{~min}, n=5$ ). The $\mathrm{LT}$ efflux in the perfusate was higher at the beginning, peak and at the end of the experiments in the LPS pretreatment group (, $1 \mathrm{mg} / \mathrm{kg}$ b.w. for $3 \mathrm{~h}, n=5)$. (c) Kupffer cell activation by Zymosan A $(150 \mu \mathrm{g} / \mathrm{ml}, 40-46 \mathrm{~min}, n=5)$ in BDL animals increased portal perfusion pressure. Intraperitoneal LPS administration (1 mg/kg b.w. for $3 \mathrm{~h}, n=5)$ increased basal, maximal, and chronic portal perfusion pressures $\left({ }^{*} P<0.05,{ }^{\sharp} P<0.05,{ }^{\circledR} P<0.05\right)$. In the same experimental setting, the additional infusion of the leukotriene $C_{4} / D_{4}$ receptor antagonist Ly171883 $(20 \mu \mathrm{M}, 30-60 \mathrm{~min}, n=5)$ or of the thromboxane $A_{2}$ receptor antagonist BM 13.177 (20 $\mu \mathrm{M}, 30-60$ min, $n=5)$ attenuated the acute $\left({ }^{\# \#} P<0.05,{ }^{\# \# \#} P<0.05\right)$ and the chronic $\left({ }^{\S} P<0.05,{ }^{\S \S} P<0.05\right)$ portal perfusion pressure increases. The additional administration

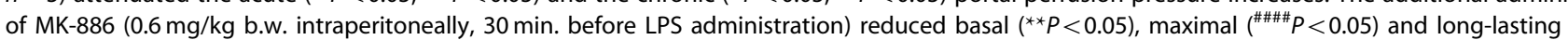
$\left({ }^{(\$ \$ \$} P<0.05\right)$ portal perfusion pressure increases. 

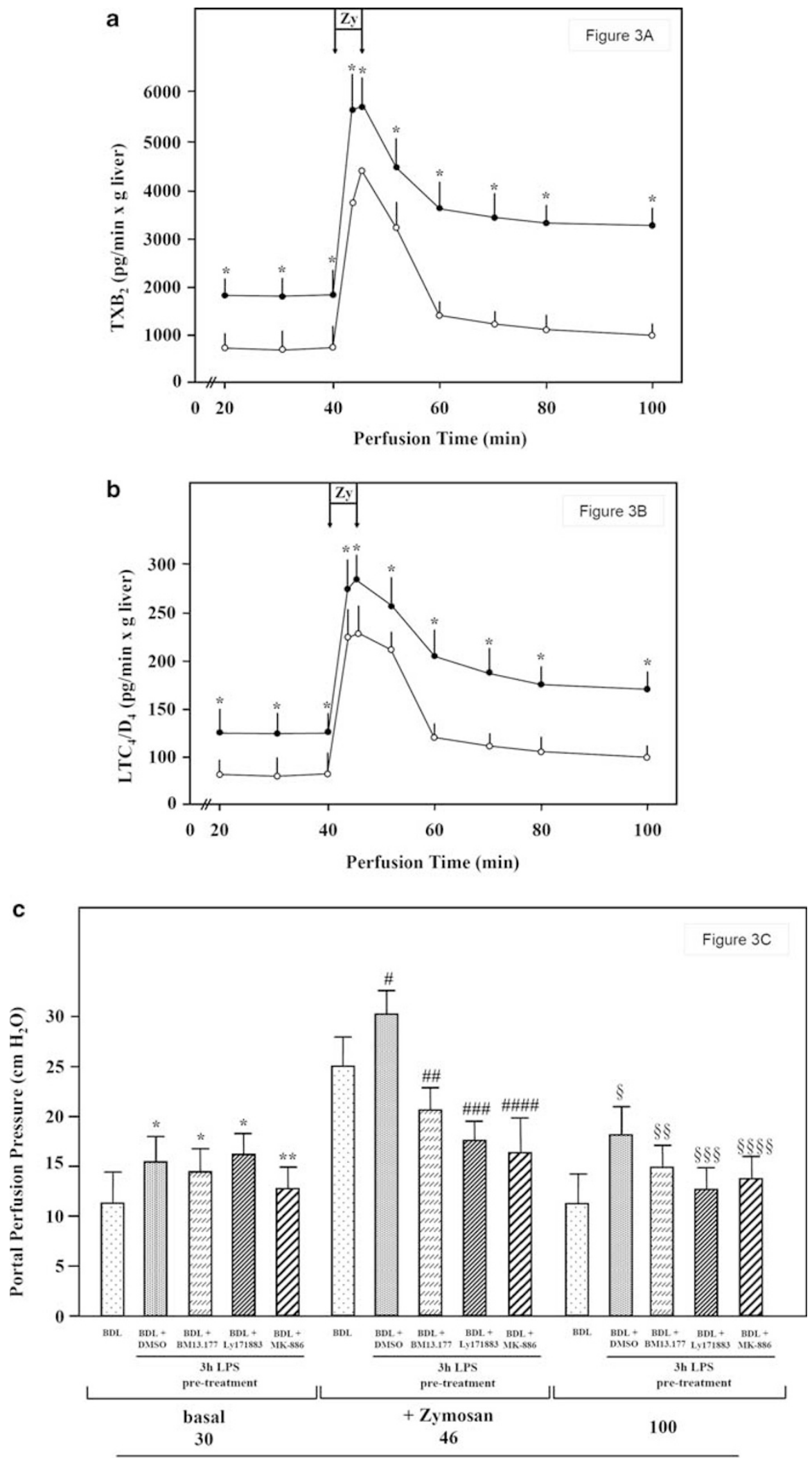

Time Point Portal Perfusion Pressure Measurement (min) 


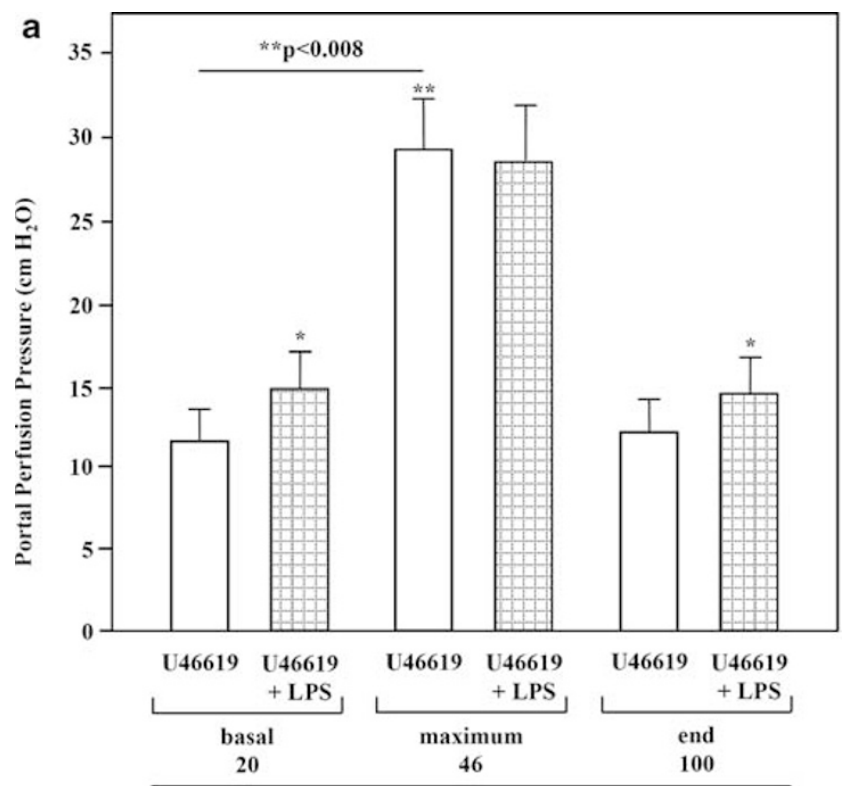

Time Point Portal Perfusion Pressure Measurement (min)

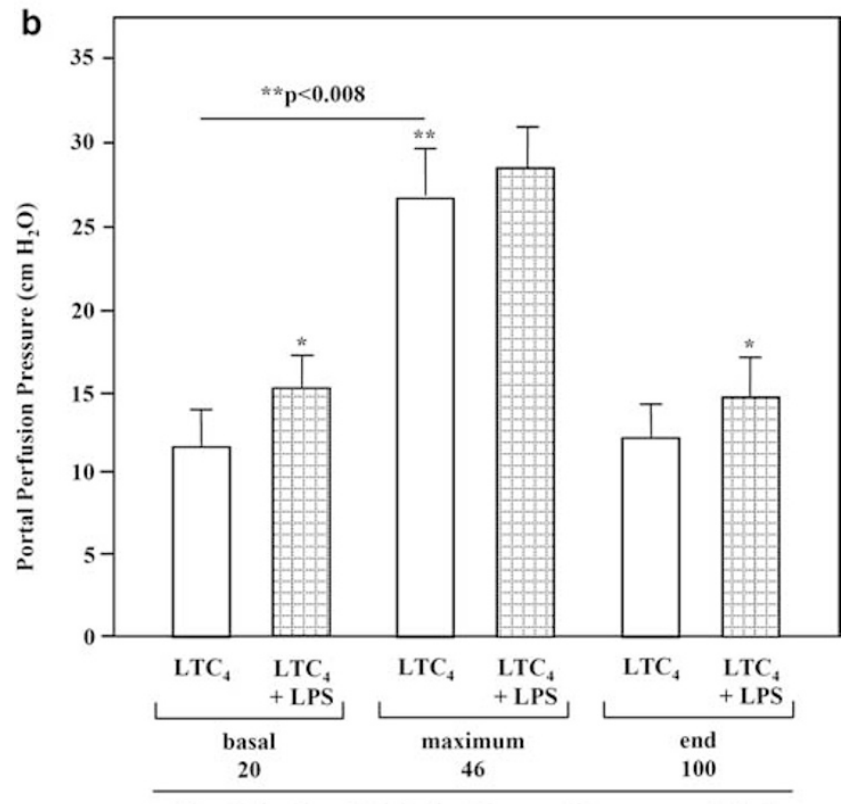

Time Point Portal Perfusion Pressure Measurement (min)

Figure 4 Intraperitoneal LPS did not alter response to the vasoconstrictors. Data are expressed as mean \pm s.d. (a) Infusion of the TX analog U46619 $(0.1 \mu \mathrm{M}, 40-46 \mathrm{~min}, n=5)$ increased portal perfusion pressure $\left({ }^{* *} P<0.008\right)$ in biliary fibrosis, which was not altered by intraperitoneal LPS pretreatment ( $1 \mathrm{mg} / \mathrm{kg}$ b.w. for $3 \mathrm{~h}, n=5$ ). (b) Again, infusion of $\mathrm{LTC}_{4}(20 \mathrm{~nm}, 40-46 \mathrm{~min}$, $n=5)$ elevated portal perfusion pressure $\left({ }^{*} P<0.008\right)$, and intraperitoneal LPS administration ( $1 \mathrm{mg} / \mathrm{kg}$ b.w. for $3 \mathrm{~h}, n=5$ ) did not change the portal perfusion pressure increase.

decreased slightly, but not significantly, from the intraperitoneal LPS pretreatment (data not shown). The additional intraportal administration of LPS did not affect the mean arterial pressure, whereas zymosan infusion decreased the mean arterial pressure (data not shown).
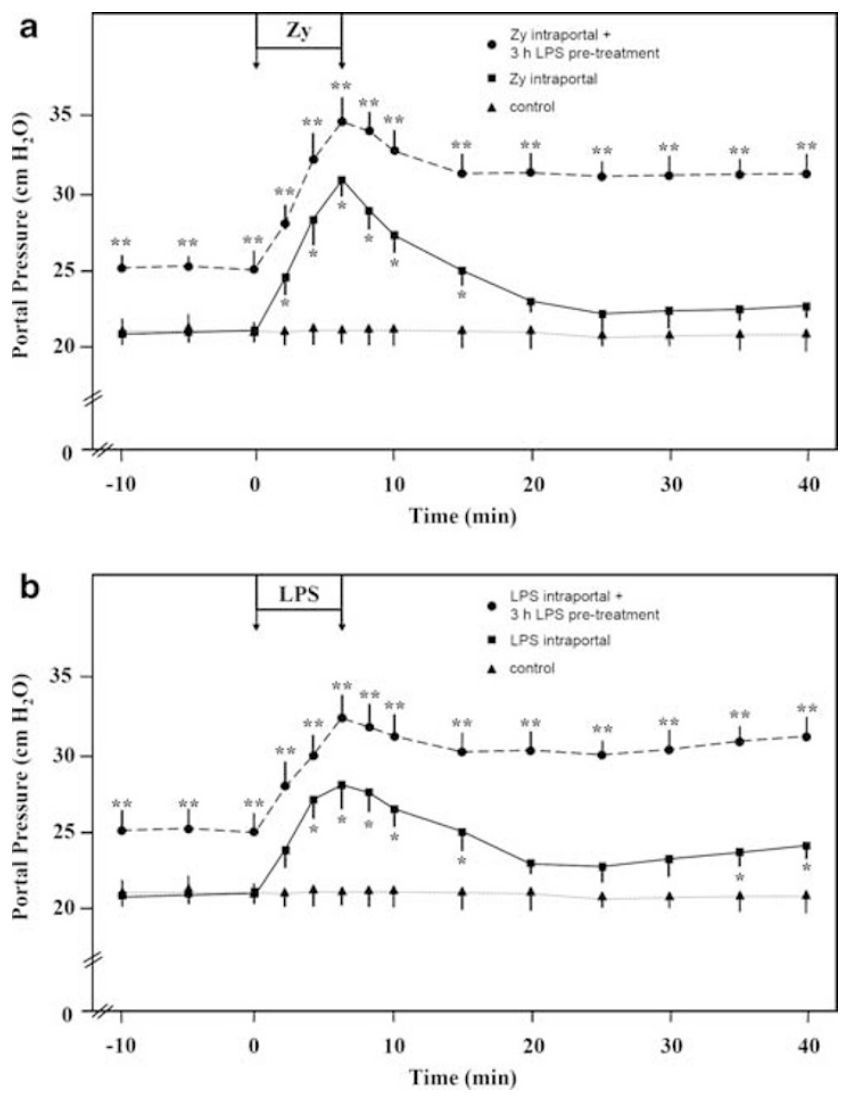

Figure 5 In vivo effects of intraperitoneal LPS for $3 \mathrm{~h}$ and additional intraportal zymosan or LPS administration. Data are expressed as mean \pm s.d. (a) Intraperitoneal LPS administration $(\bullet, 1 \mathrm{mg} / \mathrm{kg}$ b.w. for $3 \mathrm{~h}$, $n=5$ ) extended the increase in portal pressures from transient to chronic in vivo after intraportal zymosan infusion ( $, Z y, 3.2 \mathrm{mg} / \mathrm{min}, 0-6 \mathrm{~min}, n=7$, $\left.{ }^{*} P<0.05\right)$. Control experiments with a stabilization period of $10 \mathrm{~min}$ and a observation period of $40 \mathrm{~min}$ afterwards showed no change in portal pressure $(\mathbf{\Lambda}, n=4)$. (b) Subsequent to the intraperitoneal LPS pretreatment for $3 \mathrm{~h}(1 \mathrm{mg} / \mathrm{kg}$ b.w.), LPS was additionally infused through the portal vein in fibrotic livers during in vivo experiments $\left(\bullet,{ }^{* *} P<0.05, n=5\right)$. Basal portal pressure, maximal portal pressure and portal pressure at the end of the observation period of $40 \mathrm{~min}$ were augmented compared with the experiments without LPS pretreatment $(\boldsymbol{\square}, n=5)$. Intraportal LPS infusion alone $\left(\boldsymbol{\square},{ }^{\star} P<0.05, n=5\right)$ increased portal pressure acutely and resulted in a portal pressure increase at the end of the observation period of $40 \mathrm{~min}$.

\section{DISCUSSION}

This study described a novel mechanism of portal pressure increase in fibrotic livers by intraperitoneal LPS administration. There were three principal findings of our study: (1) The intraperitoneal administration of LPS for $3 \mathrm{~h}$ increased basal portal pressure and extended the portal pressure increase that occurred after a second administration of microbial constituents zymosan or LPS from transient to long lasting; (2) The hepatic expression of TLR4 and MyD88 was increased after LPS pretreatment, and the LPS-pretreated livers responded with an augmented and relevant efflux of vasoconstrictors TX and LT; and, (3) In contrast, LPS pretreatment did not alter the responsiveness to TX or $\mathrm{LTC}_{4}$. These results were obtained using in situ-perfused livers 
confirmed by subsequent in vivo studies. We investigated the effects in sham-operated and bile duct-ligated rats. The diagnosis of fibrosis was made on the basis of the presence of ascites and elevated portal pressure in vivo.

\section{Effects of LPS Pretreatment on Portal Pressure}

The initial set of experiments showed that basal portal perfusion pressure in situ and portal pressure in vivo were higher in BDL rats than in sham-operated rats. The intraperitoneal application of LPS for $3 \mathrm{~h}$ further increased basal portal pressure and extended the portal pressure increase caused by the administration of zymosan or LPS from transient to long lasting. This effect was observed in fibrotic animals 4 weeks after BDL. Intraperitoneal LPS did not affect portal perfusion pressure in sham-operated animals.

The experimental setting may be of clinical relevance. Patients with liver cirrhosis often suffer from SBP. To simulate SBP, we applied intraperitoneal LPS in cirrhotic rats. Cirrhotic rats are characterized by bacterial translocation. ${ }^{17,23}$ Interestingly, the intraperitoneal application of bacterial endotoxin affected portal pressure and enhanced the response to the microbial constituents that reached the liver through the portal vein. Different routes of bacterial constituents into the portal vein as a 'second hit' are conceivable. First, bacterial products may reach the systemic blood flow as a consequence of the SBP and thereby reach the liver. Second, patients with liver cirrhosis have increased bowel permeability and decreased bowel motility, which can result in a bacterial contamination of the portal vein by translocation of gut bacteria to mesenteric lymph nodes. ${ }^{24}$ Finally, patients with liver cirrhosis have an impaired immune system and may therefore frequently suffer from bacterial infections.

\section{KC-Dependent Effects of Intraperitoneal LPS}

The simultaneous treatment with the KC-depleting agent $\mathrm{GdCl}_{3}$ reduced the basal, maximal and long-lasting increases of portal perfusion pressure induced by LPS pretreatment. This indicates that LPS effects were mainly mediated by KCs. $\mathrm{GdCl}_{3}$ has been used in different studies to block KCs. ${ }^{25-27}$ The KC-dependent and long-lasting increase in portal perfusion pressure subsequent to LPS pretreatment was in accordance with earlier studies. ${ }^{5}$ In this previous study, the basal, maximal and long-lasting portal perfusion pressures were increased by additional LPS pretreatment for $6 \mathrm{~h}$ in prefibrotic rats 1 week after BDL. In this study, the livers had a pronounced fibrosis 4 weeks after BDL, and portal hypertension was determined both in situ and in vivo. The additional intraportal administration of two different microbial products, $\beta$-glycan-rich zymosan and LPS, was studied. As the portal pressure response to these constituents was enhanced subsequent to intraperitoneal LPS application for $3 \mathrm{~h}$, an altered disposition of the involved cells was postulated as a consequence of LPS pretreatment. This hypothesis was tested by western blot analyses from whole liver homogenates. The protein expressions of TLR4 and its effector MyD88 were increased in BDL livers when compared with sham-operated livers in a previous study. ${ }^{28}$ Interestingly, LPS pretreatment for $3 \mathrm{~h}$ further upregulated the expression of TLR4 and MyD88 in BDL livers in this study. This LPSinduced increase was attenuated by $\mathrm{GdCl}_{3}$ pretreatment. TLR4 and MyD88 are expressed on KCs, sinusoidal endothelial cells and hepatic stellate cells. ${ }^{15,29,30}$ KCs are highly relevant for the production of vasoconstrictors in the intrahepatic microvasculature. ${ }^{6,16}$ The effects observed in this study seem to be predominantly dependent on the KCs, because $\mathrm{GdCl}_{3}$ pretreatment attenuated TLR4 and MyD88 expression after LPS pretreatment.

\section{Vasoconstrictor Production and Responsiveness after LPS Pretreatment}

The cells most affected by intrahepatic vasoconstrictors are the activated hepatic stellate cells and myofibroblasts. ${ }^{31,32}$ Therefore, we next examined the production of the vasoconstrictors and the response to them after intraperitoneal LPS pretreatment. LPS pretreatment augmented the generation of two relevant vasoconstrictors: TX and the Cys-LTs. The augmented production of TX by LPS pretreatment has been described previously, ${ }^{33}$ whereas increased LT generation after intraperitoneal LPS has not been described, to our knowledge. The effects of antagonists for the TX receptor and the LT receptor, as well as the effect of the LT synthase blocker on portal perfusion pressure demonstrated the functional relevance of the enhanced production of the vasoconstrictors. The importance of the TX receptor for hepatic microcirculation has been described previously. ${ }^{6,34}$ As shown by our study, LTs and the reaction with their receptors may be of similar relevance in the intrahepatic microvasculature. The activity of IL-6 production, eg, by KCs in the liver, was not influenced by the LT synthase inhibitor MK-886. In contrary, inhibition of LT synthesis attenuated portal perfusion pressure increase. Importantly, they have the advantage, that they have no adverse effects on blood coagulation. This study showed that the infusion of the TX analog U46619 and the direct infusion of LTC $_{4}$ both increased portal perfusion pressure, as described previously. ${ }^{35}$ It is noteworthy that this response was not altered by the existence of intraperitoneal LPS in BDL animals. An enhanced vasoconstrictor response has been described previously in different models of liver cirrhosis, eg, by the upregulation of the intrahepatic Rho kinase. ${ }^{31}$ However, the intraperitoneal administration of LPS for $3 \mathrm{~h}$ did not alter the intrahepatic vasoconstrictor response in BDL livers.

\section{Clinical Considerations}

Taken together, our data may propose a new model for the role of LPS in portal hypertension and variceal bleeding. We assume that chronic confrontation with LPS due to bacterial translocation from the gut to mesenteric lymph nodes may upregulate TLR4 and Myd88 expression in cirrhosis, which was further upregulated by additional intraperitoneal LPS 
application. This confers hypersensitivity to LPS. As LPS may increase portal pressure by the production of intrahepatic vasoconstrictors including LTs, a sudden massive confrontation with LPS in SBP or infections may lead to severe aggravation of portal hypertension and variceal bleeding. Therefore, LT receptor antagonists may represent a promising treatment option in addition to early administration of antibiotics in SBP.

\section{Conclusions}

In conclusion, intraperitoneal LPS leads to the production of vasoconstrictors in the liver, including TX and LTs. As the expression of TLR4 and its effector MyD88 is increased in fibrotic livers, this confers hypersensitivity to LPS. In addition to antibiotics, LT inhibitors could therefore be an interesting therapeutic option in chronic liver diseases.

\section{ACKNOWLEDGEMENTS}

We thank Ingrid Liss for her excellent technical assistance and Enrico de Toni for careful reading of this paper. We also thank Frigga Beitinger from the Institute of Pathology of the University of Munich for the preparation and analyses of the HE and EvG staining, as well as Peter Dirschedl for his support in the statistical preparation and analysis of this study. This study is part of the thesis of Anna C Hartmann. This study was supported by grants from the Faculty of Medicine, University of Munich (MolMed and LebMit) and by the Deutsche Forschungsgemeinschaft (DFG, STE 1022/2-1).

\section{DISCLOSURE/CONFLICT OF INTEREST}

The authors declare no conflict of interest.

1. Moller S, Bendtsen F, Henriksen JH. Splanchnic and systemic hemodynamic derangement in decompensated cirrhosis. Can J Gastroenterol 2001;15:94-106.

2. Wiest R, Tsai MH, Groszmann RJ. Octreotide potentiates PKC dependent vasoconstrictors in portal-hypertensive and control rats. Gastroenterology 2001;120:975-983.

3. Hennenberg M, Trebicka J, Stark C, et al. Sorafenib targets dysregulated Rho kinase expression and portal hypertension in rats with secondary biliary cirrhosis. Br J Pharmacol 2009;157:258-270.

4. Pinzani M, Vizzutti F. Fibrosis and cirrhosis reversibility: clinical features and implications. Clin Liver Dis 2008;12:901-913, x.

5. Miller AM, Masrorpour M, Klaus C, et al. LPS exacerbates endothelin-1 induced activation of cytosolic phospholipase $\mathrm{A} 2$ and thromboxane $\mathrm{A} 2$ production from Kupffer cells of the prefibrotic rat liver. J Hepatol 2007;46:276-285.

6. Steib CJ, Gerbes AL, Bystron M, et al. Kupffer cell activation in normal and fibrotic livers increases portal pressure via thromboxane $A(2)$. J Hepatol 2007;47:228-238.

7. Graupera M, Garcia-Pagan JC, Titos E, et al. 5-Lipoxygenase inhibition reduces intrahepatic vascular resistance of cirrhotic rat livers: a possible role of cysteinyl-leukotrienes. Gastroenterology 2002;122:387-393.

8. Wiest R, Groszmann RJ. The paradox of nitric oxide in cirrhosis and portal hypertension: too much, not enough. Hepatology 2002;35:478-491.

9. Bendtsen F, Krag A, Moller S. Treatment of acute variceal bleeding. Dig Liver Dis 2008:40:328-336.

10. Bernard B, Cadranel JF, Valla D, et al. Prognostic significance of bacterial infection in bleeding cirrhotic patients: a prospective study. Gastroenterology 1995;108:1828-1834.

11. Goulis J, Patch D, Burroughs AK. Bacterial infection in the pathogenesis of variceal bleeding. Lancet 1999;353:139-142.

12. Borzio $M$, Salerno F, Piantoni $L$, et al. Bacterial infection in patients with advanced cirrhosis: a multicentre prospective study. Dig Liver Dis 2001;33:41-48.
13. Hou MC, Lin HC, Liu TT, et al. Antibiotic prophylaxis after endoscopic therapy prevents rebleeding in acute variceal hemorrhage: a randomized trial. Hepatology 2004;39:746-753.

14. Steib CJ, Gerbes AL. Signaling pathways in liver diseases: Kupffer cells. In: Dufour J-F, Clavien P-A (eds). Signaling Pathways in Liver Diseases. Springer Verlag: Heidelberg, 2009.

15. Bilzer M, Roggel F, Gerbes AL. Role of Kupffer cells in host defense and liver disease. Liver Int 2006;26:1175-1186.

16. Xu H, Korneszczuk K, Karaa A, et al. Thromboxane A2 from Kupffer cells contributes to the hyperresponsiveness of hepatic portal circulation to endothelin-1 in endotoxemic rats. Am J Physiol Gastrointest Liver Physiol 2005;288:G277-G283.

17. Neugebauer $H$, Hartmann $P$, Krenn $S$, et al. Bacterial translocation increases phagocytic activity of polymorphonuclear leucocytes in portal hypertension: priming independent of liver cirrhosis. Liver Int 2008;28:1149-1157.

18. Garcia-Tsao G, Wiest R. Gut microflora in the pathogenesis of the complications of cirrhosis. Best Pract Res Clin Gastroenterol 2004;18:353-372.

19. Hennenberg $M$, Trebicka J, Biecker $E$, et al. Vascular dysfunction in human and rat cirrhosis: role of receptor-desensitizing and calciumsensitizing proteins. Hepatology 2007;45:495-506.

20. Bilzer M, Paumgartner G, Gerbes AL. Glutathione protects the rat liver against reperfusion injury after hypothermic preservation. Gastroenterology 1999;117:200-210.

21. Schauer RJ, Gerbes AL, Vonier D, et al. Induction of cellular resistance against Kupffer cell-derived oxidant stress: a novel concept of hepatoprotection by ischemic preconditioning. Hepatology 2003;37:286-295.

22. Bilzer M, Baron A, Schauer R, et al. Glutathione treatment protects the rat liver against injury after warm ischemia and Kupffer cell activation. Digestion 2002;66:49-57.

23. Tazi KA, Moreau R, Herve $P$, et al. Norfloxacin reduces aortic NO synthases and proinflammatory cytokine up-regulation in cirrhotic rats: role of Akt signaling. Gastroenterology 2005;129:303-314.

24. Garcia-Tsao G, Wiest R. Gut microflora in the pathogenesis of the complications of cirrhosis. Best Pract Res Clin Gastroenterol 2004;18:353-372.

25. Krohn N, Kapoor S, Enami Y, et al. Hepatocyte transplantation-induced liver inflammation is driven by cytokines-chemokines associated with neutrophils and Kupffer cells. Gastroenterology 2009;136: 1806-1817.

26. Tejima $K$, Arai $M$, Ikeda $H$, et al. Ischemic preconditioning protects hepatocytes via reactive oxygen species derived from Kupffer cells in rats. Gastroenterology 2004;127:1488-1496.

27. Joseph B, Malhi $\mathrm{H}$, Bhargava KK, et al. Kupffer cells participate in early clearance of syngeneic hepatocytes transplanted in the rat liver. Gastroenterology 2002;123:1677-1685.

28. Seki E, De Minicis S, Osterreicher CH, et al. TLR4 enhances TGF-beta signaling and hepatic fibrosis. Nat Med 2007;13:1324-1332.

29. Uhrig A, Banafsche R, Kremer M, et al. Development and functional consequences of LPS tolerance in sinusoidal endothelial cells of the liver. J Leukoc Biol 2005;77:626-633.

30. Guo J, Loke J, Zheng F, et al. Functional linkage of cirrhosis-predictive single nucleotide polymorphisms of Toll-like receptor 4 to hepatic stellate cell responses. Hepatology 2009;49:960-968.

31. Zhou Q, Hennenberg M, Trebicka J, et al. Intrahepatic upregulation of RhoA and Rho-kinase signalling contributes to increased hepatic vascular resistance in rats with secondary biliary cirrhosis. Gut 2006;55:1296-1305.

32. Trebicka J, Hennenberg $M$, Laleman $W$, et al. Atorvastatin lowers portal pressure in cirrhotic rats by inhibition of RhoA/Rho-kinase and activation of endothelial nitric oxide synthase. Hepatology 2007;46:242-253.

33. Yokoyama $\mathrm{Y}, \mathrm{Xu} \mathrm{H}$, Kresge $\mathrm{N}$, et al. Role of thromboxane $\mathrm{A} 2$ in early BDL-induced portal hypertension. Am J Physiol Gastrointest Liver Physiol 2003;284:G453-G460.

34. Katagiri $\mathrm{H}$, Ito $\mathrm{Y}$, Ito $\mathrm{S}$, et al. TNF-alpha induces thromboxane receptor signaling-dependent microcirculatory dysfunction in mouse liver. Shock 2008;30:463-467.

35. Titos E, Claria J, Bataller R, et al. Hepatocyte-derived cysteinyl leukotrienes modulate vascular tone in experimental cirrhosis. Gastroenterology 2000;119:794-805. 